\title{
Heart Arhythmia Detection using Wavelet Coherence and Firefly Algorithm
}

\author{
Padmavathi Kora, PhD \\ Gokaraju Rangaraju Institute \\ of Engineering Technology \\ Hyderabad, India
}

\author{
Ch. Usha Kumari, PhD \\ Gokaraju Rangaraju Institute \\ of Engineering Technology \\ Hyderabad, India
}

\author{
K. Meenakshi, $\mathrm{PhD}$ \\ Gokaraju Rangaraju Institute \\ of Engineering Technology \\ Hyderabad, India
}

\begin{abstract}
Atrial Fibrillation (AF) is a common type of heart abnormality. During the AF electrical discharges in the atrium are rapid that results in irregular heart beat. The morphology of ECG changes due to the abnormalities in the heart. This paper consists of three major steps for the detection of heart diseases: signal pre-processing, feature extraction and classification. Feature extraction is the key process in detecting the heart abnormality. Most of the ECG detection systems depend on the time domain features for cardiac signal classification. In this paper we proposed a Wavelet Coherence (WTC) technique for ECG signal analysis. The WTC measures the similarity between two waveforms in frequency domain. Parameters extracted from WTC function is used as the features of the ECG signal. These features are optimized using Firefly algorithm (FFA). The optimized features from the FFA are given as the input to the Levenberg Marquardt Neural Network (LM NN) classifier. From the literature it is observed that the performance of the classifier is improved with the help of the optimized (reduced) features.
\end{abstract}

\section{General Terms}

Heart Arhythmia detection using Wavelet Coherence and Firefly Algorithm

\section{Keywords}

ECG, Atrial Fibrillation, Wavelet Coherence, Firefly algorithm.

\section{INTRODUCTION}

$\mathrm{AF}$ is the widely spread, cleanically diagnosed arrhythmia in the world. In the western countries, approximately 1-5\% of general population are affected by the AF. This arrhythmia increases with age. AF causes the risk factors such as heart stroke and heart diseases.

Atrial Fibrillation (AF) is automatic repeated contraction of muscle fibers. This is caused when atria beat rapidly, chaotically and ineffectively. This is indicated by the irregular fluctuations in the baseline of the ECG, but the QRS contours are usually normal. AF occurs as a consequence of rheumatic disease, atherosclerotic disease, hyperthyroidism and pericarditis.

It can be detected by monitoring with regular ECG recordings. However, the clear indication of AF is the absence of ' $\mathrm{P}$ waves 'and $\mathrm{RR}$ interval variability.

Since AF beats can be asymptomatic, medical practitioners sometimes have to depend on incidental examinations of AF on the ECG. It would be quite inconvenient for doctors to go through the Electro-cardiogram manually, specially for the cases where the occurrence of AF beats are random and a long-term recording is required to detect the abnormal activity for further processing. Waveform changes in the AF

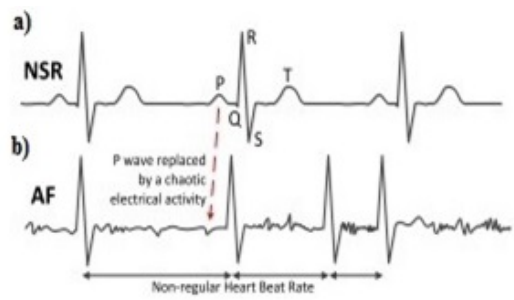

Fig. 1. Normal and AF signals

signal are presented in the Figure 1 Early detection of AF is significant because immediate treatment can save life of the patient. There are several methods to detect features of AF. Methods based on RR interval are proposed by 10 . and 21. $\mathrm{P}$ wave based methods are presented by [9]. According to 21] RR interval and $\mathrm{P}$ wave based methods have some limitations. Cardiac Arrhythmia detection Using Artificial Neural Networks with selected features were explained by [8. Statistical methods for feature extraction were explained by [2] and [17].

WTC technique for the detection of Inferior Myocardial Infarction was explained by [1] and developed by [3]. This technique is useful only to detect Inferior Myocardial detection and some other types of heart diseases. To apply this method ECG shall be segmented into beats. But the occurrence of $\mathrm{AF}$ beats are random and continuous monitoring 
of ECG recordings is needed with 10,20 or 30 second ECG segments.

The proposed WTC scheme has been compared with Auto Regressive (AR) method which is a popular method for feature extraction of ECG signals with respect to the performance measurement like accuracy in the final output.

Detection of AF using ECG involves four main steps: preprocessing, feature extraction, feature optimization and classification. The preprocessing step mainly concentrates in removing the noise from the signal using filters. The WTC technique is used to extract the frequency domain features. The morphological features that are extracted using traditional techniques generally yield a large number of features, and many of these might be insignificant. Therefore, the common practice is to extract key features useful in the classification. This paper presents FFA as a feature optimization method instead of using traditional 2] feature optimization techniques. Earlier studies extracted the key features using statistical and morphological techniques like morphological features and SVM 32, Multiple Instance Learning (MIL) 33 which give less accuracy in the classification of AF. Nature has inspired many researchers for developing algorithms to suit their needs. These algorithms are categorized basically into two types: one is based on evolutionary approach and the other is based on swarm intelligence (SI). Evolutionary algorithms mimic the Darwinian principle of " survival of the fittest" using processes of natural selection, recombination and mutation. Swarm optimization is based on the collective behavior of a group of animals. The algorithm first finds the global minimum and then searches for the best local minimum. Examples for the nature inspired optimization algorithms are Ant Colony Optimization [26, Particle Swarm Optimization 24, Bacterial Foraging Optimization (BFO) 25], 23], Bat algorithm [11, and Firefly Algorithm 29], 30]. FFA is a special class of swarm intelligence used for extracting a reduced feature set. AF signals are easily classified using Neural Networks by taking features from wavelet coherence function. WTC coefficients are the simplest and the best features for AF detection. In this paper the use of wavelet coherence function to differentiate the normal and AF signals are studied. Rest of the paper is organized as follows.

In section II, we discussed about data collection and noise removal. In section III feature extraction using Auto-regressive modeling, WTC analysis was given. In section IV feature optimization using FFA was explained. In section V classification of features by LMNN was given. In section VI simulation results are demonstrated. In section VII discussion with previous researchers was given. Finally, conclusion was given in the section VIII. The detection flow digram for AF detection is shown in Figure 2

\section{PRE PROCESSING}

\subsection{Data}

The data for the classification is taken from MIT BIH Atrial Fibrillation Database (26 patients) and Normal Sinus Rhythm Database (18 patients) of one hour duration. The first step in our algorithm is dividing the signal into desired length (10 seconds). The AF signals are sampled at $250 \mathrm{~Hz}$ and normal signals are sampled at $128 \mathrm{~Hz}$ sampling

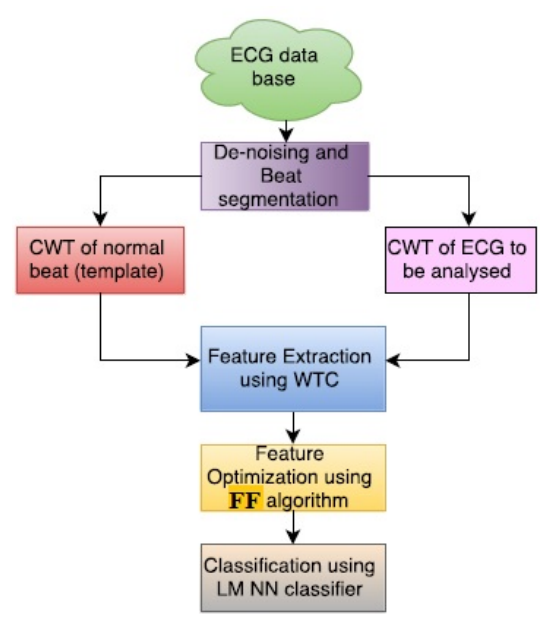

Fig. 2. Flow chart for analysis

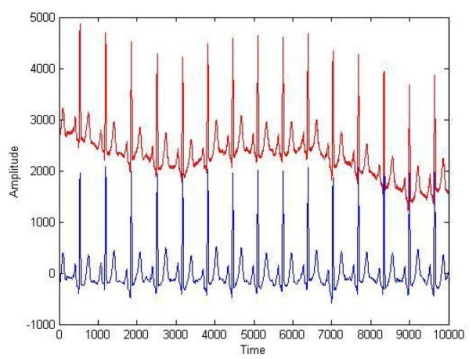

Fig. 3. Up signal:Original signal,Down signal:Baseline Wander removed signal

rate. Both the signals are re-sampled at a sampling rate of $100 \mathrm{~Hz}$ for easy comparison.

\subsection{Noise Removal}

Sgolay FIR filtering is used to remove the baseline wander present in the signal as shown Figure 3

\section{ECG FEATURE EXTRACTION USING WAVELET COHERENCE (WTC)}

\subsection{Continuous Wavelet Transform (CWT)}

A CWT is used to cut a continuous time signal into wavelets. The continuous wavelet transform has the capability to build the time frequency localization of a signal that offers good time frequency resolution. The CWT of a continuous function $\mathrm{f}(\mathrm{t})$ at a scale $\mathrm{c} 1$ and translational value $\mathrm{c} 2$ is calculated by the expression given below

$$
\begin{gathered}
F_{w}(c 1, c 2)=\frac{1}{|c 1|} \int_{-\infty}^{\infty} f(t) \bar{\psi}\left(\frac{t-c 2}{c 1}\right) \\
\psi(t)-\text { mother wavelet. }
\end{gathered}
$$

The CWT compares the signal in the form of stretched or compressed forms of a wavelet. The signal is converted into 
of wavelet at different scales and positions. CWT coefficients can be obtained by making a variation in the value of scale and position.

\subsection{Wavelet Coherence (WTC)}

Wavelet spectrum of each signal is defined by modulus and the phase of the CWT, calculated using complex valued wavelet. The individual wavelet spectra is denoted as $\mathrm{W}$ (x1) and $\mathrm{W}(\mathrm{x} 2)$. The wavelet cross-spectrum $\mathrm{W}(\mathrm{x} 1, \mathrm{x} 2)$ is taken into consideration in order to examine the time scale relation between the two signals.

$$
W(x 1, x 2)=\overline{W(x 1)} W(x 2)
$$

The magnitude of the wavelet coherence can be taken as the absolute value of the local covariance between the two time series in the time scale plane.

$$
W T C(x 1, x 2)=\frac{S(W(x 1, x 2))}{\sqrt{S\left(|W(x 1)|^{2}\right)} \sqrt{S\left(|W(x 2)|^{2}\right)}}
$$

where ' $\mathrm{S}$ ' is a smoothing operator. The wavelet coherence is analogous to the local squared correlation coefficient in time scale plane. WTC represents the variance of a time-series at different frequencies. Estimating the power spectrum over time, records the changes that happen in time scale plane, and hence termed as wavelet power spectrum. WTC is represented by various colors, where, the power is determined by the color, which ranges from blue (low power) to red (high power). Higher the power, the more similar the signals. WTC can also be interpreted as localized correlation coefficient in the time-frequency domain. It is measure of correlation/similarity between two signals at every instant in the time-frequency domain where the phase-difference indicates the relation between the signals at that instant. ECG signal of a healthy person is considered as Normal template. We have calculated the WTC of two ECG signals (Normal template and AF signal) of 10 seconds duration using the 'db4' complex wavelet for integer scales from 1 to 100. WTC is calculated for

-Normal template-Abnormal signal.

- Normal template-Normal signal.

WTC value varies between 0 to 1 . To calculate WTC, AR1 process developed by [16 is used. There are 100 rows (Scales) each row having 1000 features. The mean of the WTC values (100 rows) is calculated. It is observed from the Figure 10 that WTC values of Normal template to Normal signal have higher values than Normal template to Abnormal signals. WTC values for Normal template to all the remaining segments (Normal and Abnormal) are calculated. The Figures below show differences, when Normal to Normal, Normal to Abnormal pairs are subjected to CWT, WTC analysis:

\section{OPTIMIZATION ECG FEATURES USING FIREFLY ALGORITHM (FFA)}

The WTC method generally yields a large number of features (1000), and many of these might be insignificant. Therefore, the effective technique in this study is to extract the key features useful in the classification of ECG signals. Firefly Algorithm (FFA) can be used for two purposes: i) Calculating the optimum value of a function

ii) Reducing feature set of a population.

Here we are using the FA for the reducing the features. In this sub-section, 100 key features from 1000 samples are extracted using FFA algorithm.

This algorithm was designed by a mathematician X.S.Yang in the year 2007. FFA was formulated by mimicking the flashing (mating) activity of fireflies. Even though this algorithm is similar to the PSO [ $[$ ], Artificial Bee Colony(ABC) Optimization 4 and Ant Colony Optimization (ACO) 26], proved to be much simpler in algorithm implementation. Fireflies are small insects, which are capable of producing light to attract a prey (mate). They release small rhythmic light flashes. The light intensity attraction 'I' of fireflies decreases with the distance ' $r$ '. Hence, most fireflies are visible only up to several hundreds of meters. To execute this algorithm the fitness function is articulated based on the fluorescence light behavior of fireflies. For simplicity, it is imagined that light intensity attractiveness of firefly is determined by its brightness 'I' which is in turn connected with the fitness function.

\subsection{Attractiveness and Light Intensity}

At a particular position 'r', the brightness 'I' of a firefly can be chosen as I (r), proportional to the fitness, for a maximization problem. So the I ( $\mathrm{r}$ ) varies according to the well known inverse square law.

$$
I(r)=\frac{I s}{r^{2}}
$$

Fireflies attractiveness $\beta$ is proportional to the $\mathrm{I}(\mathrm{r})$ seen by surrounding fireflies can be defined as

$$
\beta=\beta_{0} e^{-\gamma r^{2}}
$$

where $\gamma$ is the light absorption coefficient.

\subsection{Distance}

The distance between any 2 fireflies is estimated using the distance formula. Firefly ' $\mathrm{i}$ ' is moved towards brighter firefly ' $\mathrm{j}$ ' and its movement is calculated by

\subsection{Movement}

$$
x_{i}=x_{i}+\beta_{0} e^{-\gamma r_{i, j}^{2}}\left(x_{i}-x_{j}\right)+\alpha \epsilon_{i}
$$

$$
x_{i}=x_{i}+\beta_{0} * \exp \left(-\gamma r_{i j}^{2}\right) *\left(x_{j-} x_{i}\right)+\alpha *\left(\text { rand }-\frac{1}{2}\right)
$$

The first term in eq (6) denotes the the current location of a firefly, the second term is used for determining the attractiveness $(\beta)$ of a firefly (attractive firefly), towards the 


\section{Pseudo code:Firefly Algorithm}

1. Generate the initial population randomly.

2. Calculate the fitness of initial population based on light intensity of fireflies.

3 . While $(\mathrm{t}<$ termination criteria is satisfied $)$

4.For $\mathrm{i}=1: \mathrm{p}$ ( $\mathrm{p}$ fireflies)

5.For $\mathrm{j}=1: \mathrm{p}$

6.Calculate light intensity(I) using Equation. (3).

7.Distance between two fireflies is calculated using Equation.(5).

8.If $(\mathrm{I}(\mathrm{i})<\mathrm{I}(\mathrm{j}))$

9. Firefly $\mathrm{i}$ is moved towards firefly $\mathrm{j}$ using Equation. (6)

10.Determine new solutions.

11.Else

12.Firefly $\mathrm{i}$ is moved randomly towards $\mathrm{j}$ using Equation. (7).

13. End If

14. End for $j$.

15. End for i.

16. End while

17.Sort the fireflies according to light intensity values of the new solution.

attractive neighboring fireflies and the third term indicates the random walk of a firefly (random part).

$$
x i=x i+\alpha(\operatorname{rand}-1 / 2)
$$

when firefly 'i' lacks the brighter firefly ' $\mathrm{j}$ ' then it will go for a random walk as in Eq. (7), in search of the best candidate, where the coefficient $\alpha$ is a randomization variable, and 'rand' is a random number consistently spread over the space $(0,1)$. The FFA flow diagram is shown in Fig. 4 The

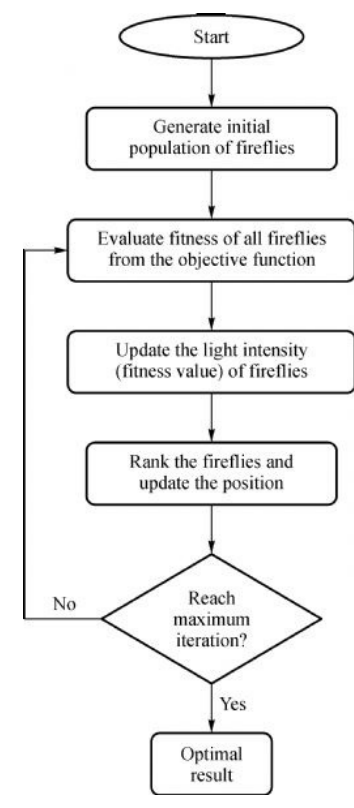

Fig. 4. FFA flowchart

pseudo code for firefly algorithm is given below The main objective of FFA feature selection stage is to reduce the features of the problem before the supervised neural network classification. Among all the wrapper algorithms used FFA, which solves optimization problems using the methods of flashing behavior of fireflies, has emerged as a promising one.

\section{RESULTS}

- CWT of Normal signal show that RR interval is regular and there is no AF (Abnormal) beats as shown in Figure 5

- CWT of Abnormal shows that RR interval is not regular and there is an AF (Abnormal) beat as shown in Figure 6 and Figure 7

-WTC of Normal-Normal shows that the two signals are highly correlated indicated by red color as shown in Figure 10

16795(NormalTemplate from MIT-BIH NSR DB)
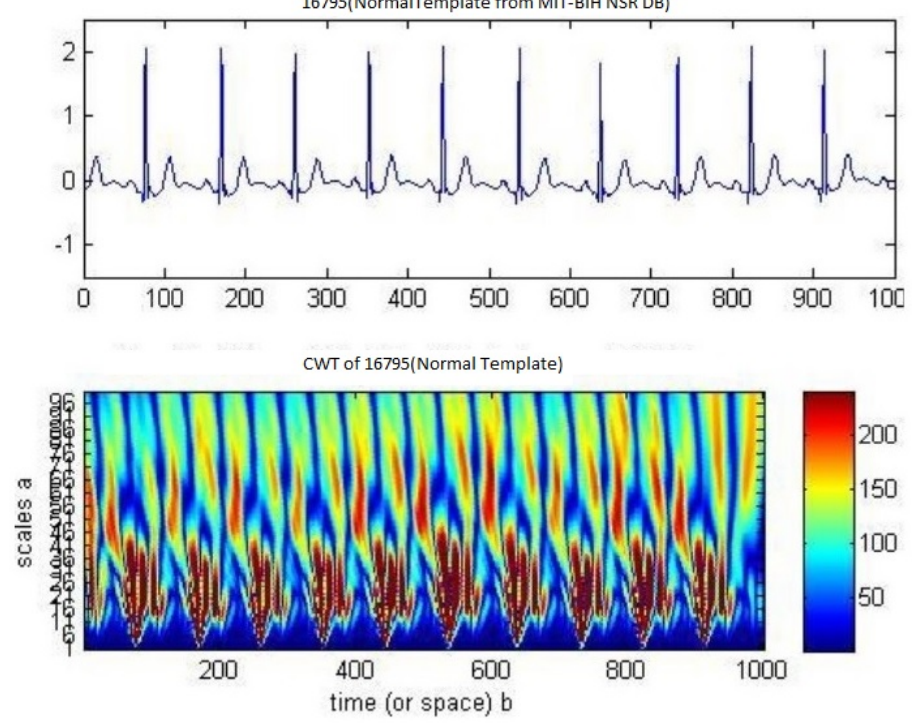

Fig. 5. CWT of Normal signal indicates that RR intervals are regular and there is no AF (Abnormal) beats
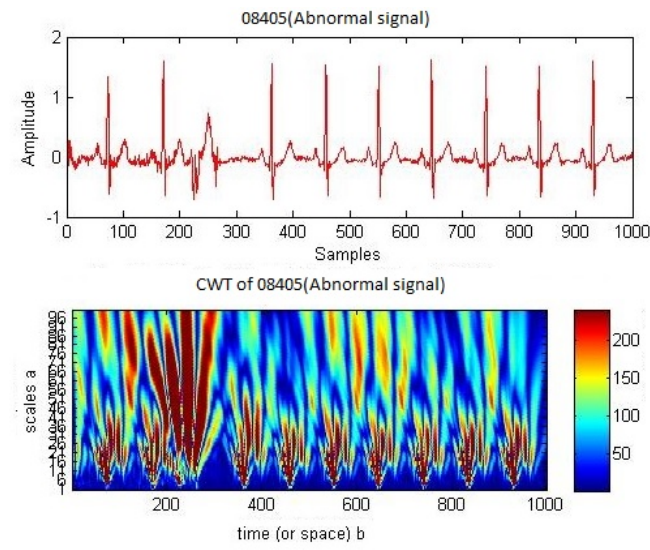

Fig. 6. CWT of Abnormal signal indicates that RR intervals are not regular and there is an AF beat in between 200-300 samples 

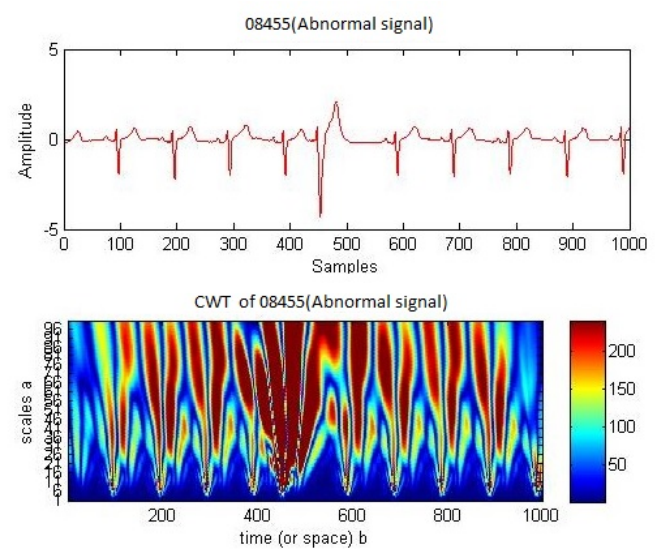

Fig. 7. CWT of Abnormal indicates that RR intervals are not regular and there is an $\mathrm{AF}$ (Abnormal) beat in between 400-500 samples.

-WTC of Normal-Abnormal shows that the two signals are uncorrelated indicated by blue color as shown in Figure 9 and Figure 8

- The results in Figure 5 Figure 6 Figure 7 depict the Heart rate irregularity of Normal and Abnormal signals.

- The results in Figure 10 Figure 9 Figure 8 depict the coherence between Normal-Normal and Normal-Abnormal signals.

- It is evident that the power decreases from red to blue. Black contour indicates the areas where power is significantly high. The Blue smudged areas indicate the lack of correlation.

- The direction of the arrows in the Figure demonstrates the correlation between two signals, wherein the arrow pointing right specifies that the two signals are in-phase implying that they are highly correlated. Arrow pointing left indicates anti-phase showing signs of uncorrelated signals. Arrows at other angles indicate that the two phenomena have a lead or lag by 90 degrees.
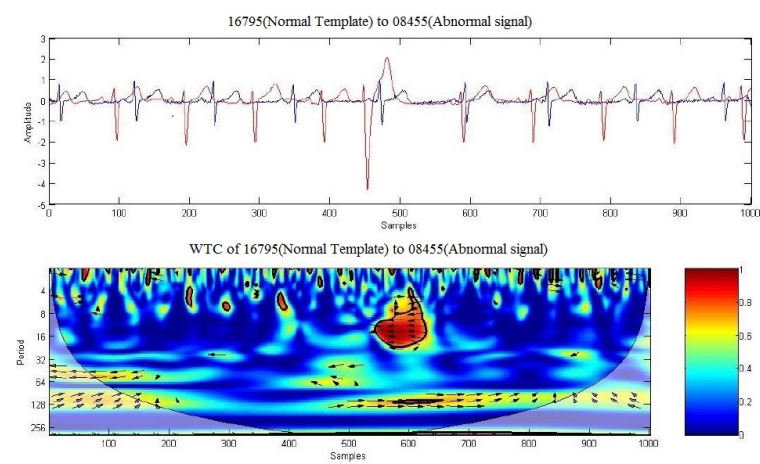

Fig. 8. WTC of Normal-Abnormal signals indicates that the two signal are highly uncorrelated indicated by large blue color portion.
Artificial Neural Network (ANN) models the nervous system of a human brain. The NN was designed to work well if it was built with 100 input neurons, 10 neurons in the hidden layer and 2 neurons in the output layer. The Levenberg
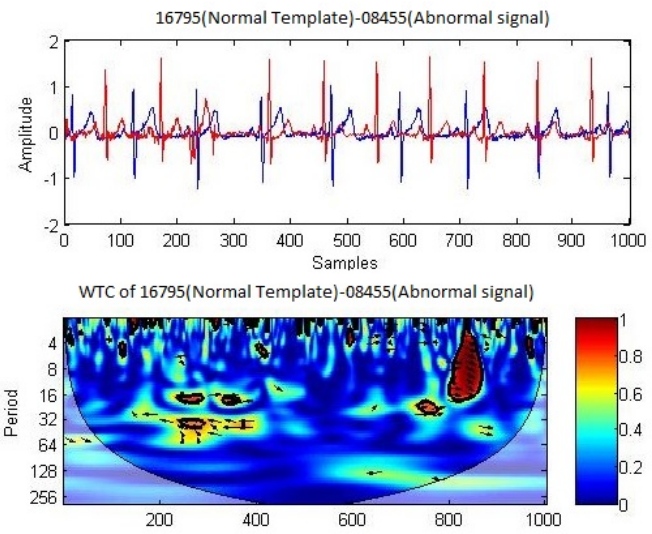

Fig. 9. WTC of Normal-Abnormal signals indicates that the two signals are highly uncorrelated indicated by large blue color portion.

Marquardt Neural Network shows good accuracy compared to SCG algorithm as shown in the table 5. To measure accuracy (Acc) two parameters sensitivity (Sen) and specificity (Spe), are calculated using the following equations.
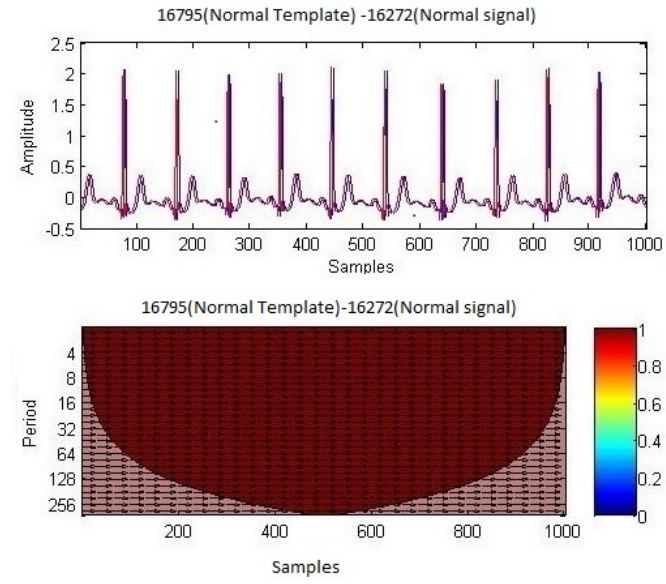

Fig. 10. WTC of Normal-Normal indicates that the two signal are highly correlated indicated by red color.

$$
\begin{gathered}
\frac{T N G}{T N G+F P G} X 100=\text { Spe } \\
\frac{T P G}{T P G+F N G} X 100=\text { Sen } \\
\frac{T P G+T N G}{T P G+T N G+F P G+F N G} X 100=A c c
\end{gathered}
$$


Table 1. Classification of features

\begin{tabular}{llll}
\hline Classifier & Sen & Spe & Acc \\
\hline AR+LM NN & $92.5 \%$ & $89.7 \%$ & $90.1 \%$ \\
AR+SCG NN & $86.5 \%$ & $83.3 \%$ & $84.4 \%$ \\
WTC+ FFA+LM & $96.97 \%$ & $99.43 \%$ & $99.1 \%$ \\
NN & & & \\
WTC+FFA+SCG & $86.4 \%$ & $99.32 \%$ & $94.07 \%$ \\
NN & & & \\
\hline
\end{tabular}

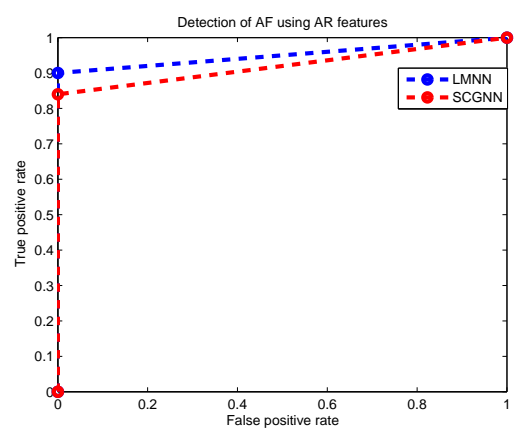

Fig. 11. AF Detection using LMNN, SCGNN with AR features

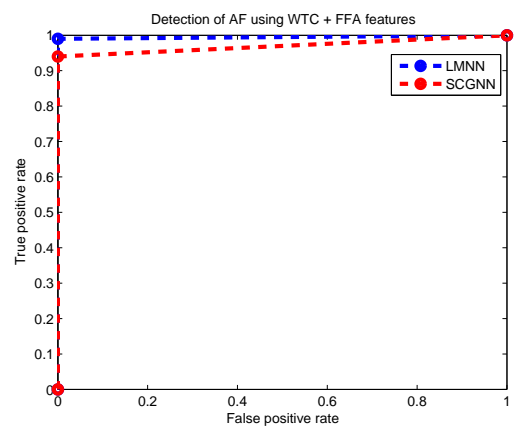

Fig. 12. AF Detection using LMNN, SCGNN with WTC features

- TPG (True Positive) $=$ Count of all the correctly classified Normal beats.

- TNG (True_Negative)=Count of all beats the correctly classified Abnormal beats.

-FPG (False_Positive)= Count of Normal beats which are classified as Abnormal.

-FNG (False_Negative) $=$ Count of Abnormal beats which are classified as Normal.

Receiver Operating Characteristic (ROC) curve is plotted for true positive rate (Sensitivity) verses false positive rate (100-Specificity) as in Fig. 11 and Fig. 12 Each point on the ROC curve represents a sensitivity/specificity pair corresponding to a particular parameter. The normal and abnormal classes can be clearly distinguished using the measure of the area under the curve.

In the training we applied multilayer NN, and checked the network performance and decide if any changes need to be made to the training process, or to the data sets of the network architecture. Neural network training with 'trainlm' Matlab function.
Table 2. Classification of features

\begin{tabular}{|c|c|c|c|c|}
\hline Studies & Approach & $\operatorname{Sen}(\%)$ & $\begin{array}{l}\text { Spe } \\
(\%)\end{array}$ & $\begin{array}{l}\text { Acc } \\
(\%)\end{array}$ \\
\hline $\begin{array}{l}\text { Tateno } \\
\text { al.(2001) 21 }\end{array}$ & $\begin{array}{ll}\text { RR Inter- } \\
\text { val }\end{array}$ & 91.20 & 96.08 & - \\
\hline $\begin{array}{l}\text { parvaresh } \\
\text { al.(2011) } 19\end{array}$ & $\begin{array}{l}\text { AR coeffi- } \\
\text { cients }\end{array}$ & 96.14 & 93.20 & 90.09 \\
\hline Lee et al.(2013) 20 & $\begin{array}{l}\text { RR Inter- } \\
\text { val }\end{array}$ & 97.26 & 95.91 & - \\
\hline Zhou et al.(2014) 22 & $\begin{array}{l}\text { Symbolic } \\
\text { dynam- } \\
\text { ics \& } \\
\text { Shannon } \\
\text { entropy }\end{array}$ & 96,89 & 98.27 & 98.03 \\
\hline $\begin{array}{l}\text { Proposed } \\
\text { proach }\end{array}$ & $\begin{array}{l}\text { WTC \& } \\
\text { FFA }\end{array}$ & 96.97 & 99.43 & 99.1 \\
\hline
\end{tabular}

\section{DISCUSSION}

The proposed method is compared against other four detection algorithms as shown in the Table?? such as RR Interval (RRI), AR coefficients, Symbolic dynamics \& Shannon entropy in terms of related features selected from the original database and classification accuracy obtained from different classifiers using Matlab software.

The work in 21], explores an experimental study based on the difference between RR intervals for extracting relevant features for the detection of AF. The values of sensitivity and specificity are $94.4 \%, 97.2 \%$ respectively.

The work presented in [19, uses AR coefficients as features for classification AF using three different classifiers. AR coefficients are calculated for each 15 segments data. The values of sensitivity and specificity are $96.14 \%, 93.20 \%$ respectively. The work proposed in [20] uses three statistical methods for the detection of AF. These techniques are tested on AFDB, NSRDB datasets. The values of sensitivity and specificity, accuracy are $97.2 \%, 95.91 \%$ and $96.14 \%$ respectively.

The work proposed in 22] uses RR intervals, and it computes various operations like nonlinear or linear integer filters, symbolic dynamics and the calculation of Shannon entropy. On line analytical processing of the method can be achieved using this novel algorithm. The values of sensitivity and specificity, accuracy were are $96.89 \%, 98.27 \%$ and $98.03 \%$ respectively.

From the experiments, this work concluded that the proposed WTC technique with FFA optimization outperformed other three algorithms with selection of minimal number of relevant features using FFA and highest classification accuracy. The FFA employed to intelligently select the most relevant features that could increase the classification accuracy while ignoring noisy and redundant features.

\section{CONCLUSION}

This procedure helps to automate certain section or a part of the diagnosis and it helps the doctors or the medical community to focus on other crucial sections. The Levenberg Marquardt Neural Network (LMNN) clearly distinguishes the AF when WTC features are optimized using FFA technique. This has also increased the accuracy of diagnosis. The future modifications to the algorithm are, use of multiple variants of FFA such as, Gaussian distribution for random movement. The chaos enhanced FFA for tuning of parame- 
ters $\alpha, \beta$ and $\gamma$ seems to be a better idea for solving practical problems such as feature extraction and classification.

\section{Conflict of interest}

The authors have no conflicts of interest to declare.

Acknowledgments

The authors wish to thank Gokaraju Rangaraju Institute of Engineering and Technology for the excellent drive toward research.

\section{REFERENCES}

[1] Banerjee, S., Mitra, M. (2014). Application of cross wavelet transform for ecg pattern analysis and classification, IEEE transcations on Instrumentation and Measurement, 63, 2, 326-333.

[2] Chatterjee, H., Gupta, R., Mitra, M. (2011). A statistical approach for determination of time plane features from digitized ecg. Computers in biology and medicine, $41,5,278-284$.

[3] Grinsted, A., Moore, J. C.,Jevrejeva, S.(2004). 'Application of the cross wavelet transform and wavelet coherence to geophysical time series'.Nonlinear processes in geophysics, Vol.11 No.5/6, pp.561-566.

[4] Pal, S. K., Rai, C. S., and Singh, A. P. "Comparative study of firefly algorithm and particle swarm optimization for noisy non-linear optimization problems". International Journal of Intelligent Systems and Applications (IJISA), 4(10), 50, 2002.

[5] J. Kennedy, R. C. Eberhart, "Particle swarm optimization", IEEE International Conference on Neural Networks, Piscataway, NJ., pp.942-1948, 1995.

[6] Dorigo, Marco, and Thomas Stutzle. "The ant colony optimization metaheuristic: Algorithms, applications, and advances." Handbook of metaheuristics. Springer US, 2003. pp.250-285.

[7] Ibn Ibrahimy, M., Ahsan, R., Khalifa, O.(2013). 'Design and optimization of levenberg-marquardt based neural network classifier for emg signals to identify hand motions'.Measurement Science Review,Vol.13,No.3, pp.142-151.

[8] Mitra, M., Samanta, R. (2013). 'Cardiac arrhythmia classification using neural networks with selected features'. Procedia Technology, Vol.10 ,pp. 76-84.

[9] Mohebbi, M.,Ghassemian, H. (2008).'Detection of atrial fibrillation episodes using svm?. In Engineering in medicine and biology society,30th annual international conference of the ieee,pp. 177-180.

[10] 2014]Moody Moody, G. B., Mark, R. G. (1983). 'A new method for detecting atrial fibrillation using rr intervals'. Computers in Cardiology,Vol.10, pp. 227-230.

[11] Yang, X. S. (2011). Bat algorithm for multi-objective optimisation. International Journal of Bio-Inspired Computation, 3(5), 267-274.

[12] Sapna, S., Tamilarasi, A., Kumar, M. P. (2012). 'Backpropagation learning algorithm based on levenberg marquardt algorithm'. $C S$ and IT-CSCP,pp. 393-398.

[13] Schamroth,L.(1964). An introduction to electrocardiography.Academic Medicine,Vol.39,No.10,

[14] Singh, G., Singh, C.(2012). 'Estimation of coherence between ecg signal and eeg signal at different heart rates and respiratory rates'. International Journal of Engineering Innovative Technologies,Vol.1 ,No.2 ,pp.159163.

[15] Tateno, K., Glass, L.(2000). 'A method for detection of atrial fibrillation using rr intervals'. In Computers in cardiology 2000,pp. 391-394.

[16] Welch, P. D. (1967). 'The use of fast fourier transform for the estimation of power spectra: a method based on time averaging over short, modified periodograms'.IEEE Transactions on audio and electroacoustics, Vol.15,No.2, pp.70-73.

[17] Weng, B., Wang, J. J., Michaud, F.,BlancoVelasco, M. (2008). 'Atrial fibrillation detection using stationary wavelet transform analysis'. In Engineering in medicine and biology society, embs 2008.30th annual international conference of the ieee, pp.1128-1131.

[18] J. G. Proakis(2001), 'Digital signal processing: principles algorithms and applications',Pearson Education India.

[19] Parvaresh, Sanaz, and Ahmad Ayatollahi. "Automatic atrial fibrillation detection using autoregressive modeling." 2011 International Conference on Biomedical Engineering and Technology. 2011.

[20] Lee, Jinseok, et al. "Atrial fibrillation detection using an iPhone 4S." Biomedical Engineering, IEEE Transactions on 60.1 (2013): 203-206.

[21] Tateno, K., and L. Glass. "Automatic detection of atrial fibrillation using the coefficient of variation and density histograms of RR and ITTRR intervals." Medical and Biological Engineering and Computing 39.6 (2001): 664671.

[22] Zhou, Xiaolin, et al. "Automatic online detection of atrial fibrillation based on symbolic dynamics and Shannon entropy." Biomedical engineering online 13.1 (2014): 18.

[23] Kora, P., and Kalva, S. R. (2015). Hybrid Bacterial Foraging and Particle Swarm Optimization for detecting Bundle Branch Block. SpringerPlus, 4(1), 481.

[24] Kennedy, J. (2010). Particle swarm optimization. In Encyclopedia of Machine Learning (pp. 760-766). Springer US.

[25] Passino, K. M. (2002). Biomimicry of bacterial foraging for distributed optimization and control. Control Systems, IEEE, 22(3), 52-67.

[26] Dorigo, M., Birattari, M., Blum, C., Clerc, M., StÃijtzle, T., and Winfield, A. (Eds.). (2008). Ant Colony Optimization and Swarm Intelligence: 6th International Conference, ANTS 2008, Brussels, Belgium, September 22-24, 2008, Proceedings (Vol. 5217). Springer.

[27] Mishra, A., Agarwal, C., Sharma, A., and Bedi, P. (2014). Optimized gray-scale image watermarking using DWTâĂŞSVD and Firefly Algorithm. Expert Systems with Applications, 41(17), 7858-7867.

[28] Nasiri, J., Naghibzadeh, M., Yazdi, H. S., and Naghibzadeh, B. (2009, November). ECG arrhythmia classification with support vector machines and genetic algorithm. In Computer Modeling and Simulation, 2009. EMS'09. Third UKSim European Symposium on (pp. 187-192). IEEE. 
[29] Mishra, A., Agarwal, C., Sharma, A., and Bedi, P. (2014). Optimized gray-scale image watermarking using DWTâĂŞSVD and Firefly Algorithm. Expert Systems with Applications, 41(17), 7858-7867.

[30] Sahu, R. K., Panda, S., and Padhan, S. (2015). A hybrid firefly algorithm and pattern search technique for automatic generation control of multi area power systems. International Journal of Electrical Power and Energy Systems, 64, 9-23.

[31] Yang, X. S., and Deb, S. (2009, December). Cuckoo search via Levy flights. In Nature and Biologically Inspired Computing, 2009. NaBIC 2009. World Congress on (pp. 210-214). IEEE.

[32] Spilka, J., Chudacek, V., Kuzilek, J., Lhotska, L., and Hanuliak, M. (2010, September). Detection of inferior myocardial infarction: A comparison of various decision systems and learning algorithms. In Computing in Cardiology, 2010 (pp. 273-276). IEEE.

[33] Sun, L., Lu, Y., Yang, K., and Li, S. (2012). ECG analysis using multiple instance learning for myocardial infarction detection. Biomedical Engineering, IEEE Transactions on, 59(12), 3348-3356.

[34] Fister, I. (2013). A comprehensive review of bat algorithms and their hybridization (Doctoral dissertation, Masters thesis, University of Maribor, Slovenia). 